\title{
Could stem cells have a role in treating mental illnesses?
}

\section{Preliminary research suggests potential therapeutic benefits for several disorders}

W hile laboratory studies move forward at full speed, the clinical use of stem cells-undifferentiated cells that can develop into many different types of specialized cells-remains controversial. Presently, only unadulterated stem cells are allowed to be used in patients, and only on an experimental and investigational basis. Stem cells that have been expanded, modified, or enhanced outside of the body are not allowed to be used for clinical application in the United States at this time. In June 2021, the FDA strengthened the language of stem cell regulation, further limiting their clinical application (see https://www. fda.gov/vaccines-blood-biologics/consumers-biologics / important-patient-and-consumer-information-about-regenerative-medicine-therapies). Yet some applications, such as treatment of lymphoma or restorative knee injections, are covered by some health insurance plans, and the acceptance of stem cell treatment is growing.

In this article, I describe the basics of stem cells, and explore the potential therapeutic use of stem cells for treating various mental illnesses.

\section{Stem cells: A primer}

Human embryonic stem cells were initially investigated for their healing properties. However, the need to harvest these cells from embryos drew much criticism, and many found the process to be ethically and religiously unacceptable. This was

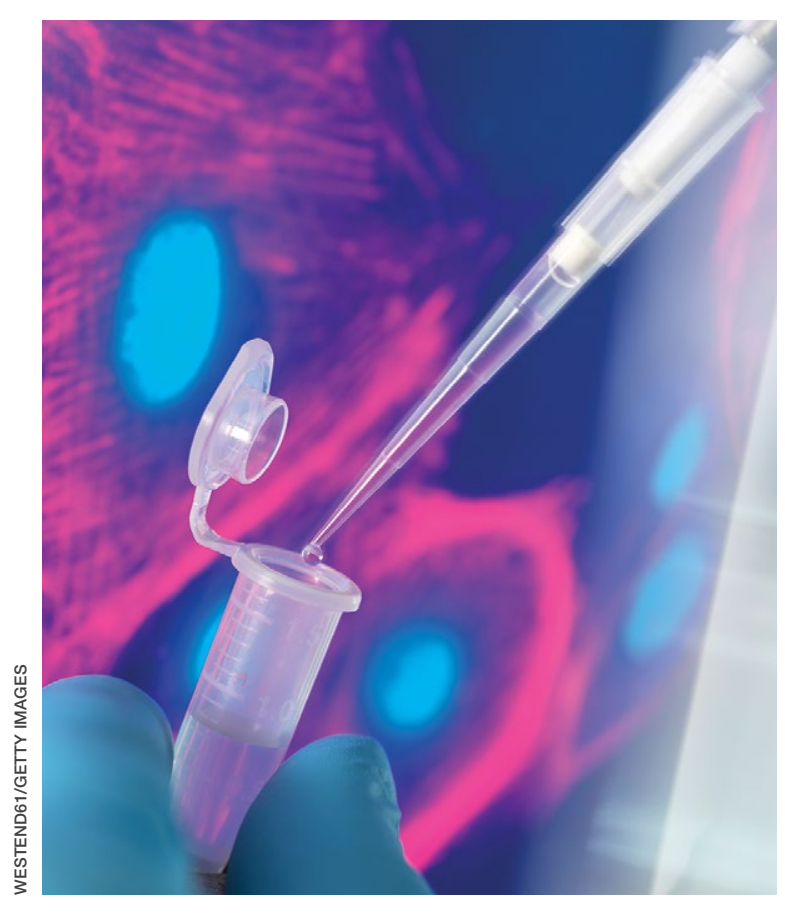

Dmitry M. Arbuck, MD

President and Medical Director Indiana Polyclinic Indianapolis, Indiana 


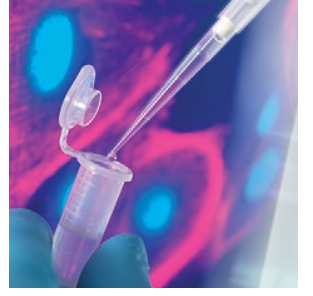

Stem cells in psychiatry

\section{Clinical Point}

The ability of stem cells to physically regenerate the CNS is directly relevant to psychiatry

Discuss this article at www.facebook.com/ MDedgePsychiatry resolved by the Nobel prize-winning discovery that adult somatic cells can be reprogrammed into cells with embryonic stem cell properties by introducing specific transcription factors. These cells have been termed "induced pluripotent stem cells" (iPSCs). ${ }^{1}$ The use of adult stem cells and stem cells from the umbilical cords of healthy newborns has allowed for wider acceptance of stem cell research and treatment.

Stem cells may be collected from the patient himself or herself; these are autologous stem cells. They may also be harvested from healthy newborn waste, such as the umbilical cord blood and wall; these are allogenic stem cells. Autologous stem cells are present in almost any tissue but are usually collected from the patient's adipose tissue or from bone marrow. Understandably, younger stem cells possess higher healing properties. Stem cells may be mesenchymal, producing primarily connective and nervous tissue, or hematopoietic, influencing the immune system and blood cell production, though there is a considerable overlap in the function of these types of cells.

Adult somatic stem cells may be turned into stem cells (iPSCs) and then become any tissue, including neurons. This ability of stem cells to physically regenerate the CNS is directly relevant to psychiatry.

In addition to neurogenesis, stem cell transplants can assist in immune and vascular restoration as well as in suppressing inflammation. The ability of stem cells to replace mutated genes may be useful for addressing inheritable neuropsychiatric conditions.

Both autoimmune and inflammatory mechanisms play an important role in most psychiatric illnesses. The more we learn, the more it is clear that brain function is profoundly dependent on more than just its structure, and that structure depends on more than blood supply. Stem cells influence the vascular, nutritional, functional, inflammatory, and immune environment of the brain, potentially assisting in cognitive and emotional rehabilitation.

Stem cells operate in 2 fundamental ways: via direct cell-to-cell interaction, and via the production and release of growth, immuneregulating, and anti-inflammatory factors.
Such factors are produced within the cells and then released in the extracellular environment as a content of exosomes. The route of administration is important in the delivery of the stem cells to the target tissue. Unlike their direct introduction into a joint, muscle, or intervertebral disk, injection of stem cells into the brain is more complicated and not routinely feasible. Intrathecal injections may bring stem cells into the CNS, but cerebrospinal fluid does not easily carry stem cells into the brain, and certainly cannot deliver them to an identified target within the brain. Existing technology can allow stem cells to be packaged in such a way that they can penetrate the blood-brain barrier, but this requires stem cell modification, which presently is not permitted in clinical practice in the United States. Alternatively, there is a way to weaken the blood-brain barrier to allow stem cells to travel through the "opened doors," so to speak, but this allows everything to have access to the CNS, which may be unsafe. IV administration is technologically easy, and it grants stem cells the environment to multiply and produce extracellular factors that can cross the blood-brain barrier, while large cells cannot.

\section{Stem cells as a treatment for mental illness}

Based on our understanding of the function of stem cells, many neurodegenerative-, vascular-, immune-, and inflammation-based psychiatric conditions can be influenced by stem cell treatment. Here I review the potential therapeutic role of stem cells in the treatment of several psychiatric disorders.

\section{Alzheimer's dementia}

Alzheimer's dementia (AD) is a progressive neurodegenerative pathology based on neuronal and synaptic loss. Repopulation and regeneration of depleted neuronal circuitry by exogenous stem cells may be a rational therapeutic strategy. ${ }^{2}$ The regeneration of lost neurons has the potential to restore cognitive function. Multiple growth factors that regulate neurogenesis are abundant during child development but dramatically decline with age. The introduction of stem cellsespecially those derived from newborn 
waste-seem to promote recovery from neurodegenerative disease or injury. ${ }^{3}$

There currently is no cure for AD. Cellular therapy promises new advances in treatment. ${ }^{4}$ Neurogenesis occurs not only during fetal development but in the adult brain. Neural stem cells reside in the adult CNS of all mammals. ${ }^{5}$ They are intimately involved in continuous restoration, but age just like the rest of the animal tissue, providing everdecreasing restorative potential.

The number of studies of stem cells in AD has increased since the early 2000s, ${ }^{6,7}$ and research continues to demonstrate robust CNS neurogenesis. In a 2020 study, Zappa Villar et $\mathrm{al}^{8}$ evaluated stem cells as a treatment for rats in which an AD model was induced by the intracerebroventricular injection of streptozotocin (STZ). The STZ-treated rats displayed poor performance in all behavioral tests. Stem cell therapy increased exploratory behavior, decreased anxiety, and improved spatial memory and marble-burying behavior; the latter was representative of daily life activities. Importantly, stem cell therapy ameliorated and restored hippocampal atrophy and some presynaptic protein levels in the rats with AD. ${ }^{8}$ Animal models cannot be automatically applied to humans, but they shine a light on the areas that need further exploration.

In humans, elevated cortisol levels during aging predict hippocampal atrophy and memory deficits, ${ }^{9}$ and this deficiency may be positively influenced by stem cell treatment.

\section{Schizophrenia}

Recent research indicates that schizophrenia may begin with abnormal neurogenesis from neural stem cells inside the embryo, and that this process may be particularly vulnerable to numerous genetic and/or environmental disturbances of early brain development. ${ }^{10}$ Because neurogenesis is not confined to the womb but is a protracted process that continues into postnatal life, adolescence and beyond, influencing this process may be a way to add to the schizophrenia treatment armamentarium. ${ }^{10}$ Sacco et $\mathrm{al}^{11}$ described links between the alteration of intrauterine and adult neurogenesis and the causes of neuropsychiatric disorders, including schizophrenia. Immune and inflammatory mechanisms are important in the etiology of schizophrenia. By their core function, stem cells address both mechanisms, and may directly modulate this devastating disease.

In addition to clinical hopes, advances in research tools hold the promise of new discoveries. With the advent of iPSC technology, it is possible to generate live neurons in vitro from somatic tissue of patients with schizophrenia. Despite its many limitations, this revolutionary technology has already helped to advance our understanding of schizophrenia. ${ }^{11}$

\section{Bipolar disorder}

Many of the fundamental neurobiological mechanisms of schizophrenia are mirrored in bipolar disorder. ${ }^{12}$ Though we are not ready to bring stem cells into the dayto-day treatment of this condition, several groups are starting to apply iPSC technology to the study of bipolar disorder. ${ }^{13}$

Neurodevelopmental factors-particularly pathways related to nervous system development, cell migration, extracellular matrix, methylation, and calcium signaling-have been identified in large gene expression studies as altered in bipolar disorder. ${ }^{14}$ Stem cell technology opens doorways to reverse engineering of human neurodegenerative disease. ${ }^{15}$

\section{Autism spectrum disorders}

Autism spectrum disorders (ASDs) are multiple heterogeneous neurodevelopmental disorders. ${ }^{16}$ Neuroinflammation and immune dysregulation influence the origin of ASDs. Due to the neurobiologic changes underlying ASD development, cell-based therapies, including the use of mesenchymal stem cells (MSCs), have been applied to ASDs. ${ }^{16}$ Stem cells show specific immunologic properties that make them promising candidates for treating ASDs. ${ }^{17}$

The exact mechanisms of action of MSCs to restore function in patients with ASDs are largely unknown, but proposed mechanisms include:

- synthesizing and releasing anti-inflammatory cytokines and survival-promoting growth factors

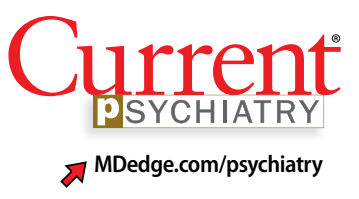

Clinical Point

Stem cell technology opens doorways to reverse engineering of human neurodegenerative disease 


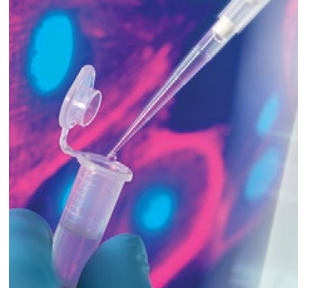

Stem cells in psychiatry

\section{Clinical Point}

\section{Small studies have} found improvements in patients with autism spectrum disorders who were treated with stem cells
- integrating into the existing neural and synaptic network

- restoring plasticity. ${ }^{18}$

In a study of transplantation of human cord blood cells and umbilical cord-derived MSCs for patients with ASDs, Bradstreet et $\mathrm{al}^{19}$ found a statistically significant difference on scores for domains of speech, sociability, sensory, and overall health, as well as reductions in the total scores, in those who received transplants compared to their pretreatment values.

In another study of stem cell therapy for ASDs, Lv et $\mathrm{al}^{20}$ demonstrated the safety and efficacy of combined transplantation of human cord blood cells and umbilical cordderived MSCs in treating children with ASDs. The transplantations included 4 stem cell IV infusions and intrathecal injections once a week. Statistically significant differences were shown at 24 weeks post-treatment. Although this nonrandomized, open-label, single-center Phase I/II trial cannot be relied on for any definitive conclusions, it suggests an important area of investigation..$^{20}$

The vascular aspects of ASDs' pathogenesis should not be overlooked. For example, specific temporal lobe areas associated with facial recognition, social interaction, and language comprehension have been demonstrated to be hypoperfused in children with ASDs, but not in controls. The degree of hypoperfusion and resulting hypoxia correlates with the severity of ASD symptoms. The damage causing hypoperfusion of temporal areas was associated with the onset of autism-like disorders. Damage of the amygdala, hippocampus, or other temporal structures induces permanent or transient autistic-like characteristics, such as unexpressive faces, little eye contact, and motor stereotypes. Clinically, temporal lobe damage by viral and other means has been implicated in the development of ASD in children and adults. Hypoperfusion may contribute to defects, not only by inducing hypoxia, but also by allowing for abnormal metabolite or neurotransmitter accumulation. This is one of the reasons glutamate toxicity has been implicated in ASD. The augmentation of perfusion through stimulation of angiogenesis by stem cells should allow for metabolite clearance and restoration of functionality. Vargas et $\mathrm{al}^{21}$ compared brain autopsy samples from 11 children with ASDs to those of 7 agematched controls. They demonstrated an active neuroinflammatory process in the cerebral cortex, white matter, and cerebellum of patients with ASDs, both by immunohistochemistry and morphology. ${ }^{21}$

Multiple studies have confirmed that the systemic administration of cord blood cells is sufficient to induce neuroregeneration..$^{22,23}$ Angiogenesis has been experimentally demonstrated in peripheral artery disease, myocardial ischemia, and stroke, and has direct implications on brain repair. ${ }^{24}$ Immune dysregulation ${ }^{25,26}$ and immune modulation ${ }^{27}$ also are addressed by stem cell treatment, which provides a promising avenue for battling ASDs.

Like attention-deficit/hyperactivity disorder and obsessive-compulsive disorder, ASDs are neurodevelopmental conditions. Advances based on the use of stem cells hold great promise for understanding, diagnosing and, possibly, treating these psychiatric disorders. ${ }^{28,29}$

\section{Depression}

Neuropsychiatric disorders arise from deviations from the regular differentiation process of the CNS, leading to altered neuronal connectivity. Relatively subtle abnormalities in the size and number of cells in the prefrontal cortex and basal ganglia have been observed in patients with depressive disorder and Tourette syndrome. ${ }^{30}$ Fibroblast-derived iPSCs generate serotonergic neurons through the exposure of the cells to growth factors and modulators of signaling pathways. If these serotonergic neurons are made from the patients' own cells, they can be used to screen for new therapeutics and elucidate the unknown mechanisms through which current medications may function. ${ }^{31}$ This development could lead to the discovery of new medication targets and new insights into the molecular biology of depression. ${ }^{32}$

Deficiencies of brain-derived neurotrophic factor (BDNF) have a role in depression, anxiety, and other neuropsychiatric illnesses. The acute behavioral effects of selective serotonin reuptake inhibitors and tricyclic antidepressants 


\section{Stem cells and substance use disorders}

$\mathbf{R}_{\mathrm{c}}^{\mathrm{s}}$ searchers have begun to explore stem cells as a potential treatment for several substance use disorders, including those involving alcohol, cocaine, and opioids, as well as their interactions with cannabinoids.

Alcohol use disorder. In a 2017 study, Israel et $\mathrm{al}^{41}$ gave intra-cerebral injections of mesenchymal stem cells (MSCs) to rats that were bred to have a high alcohol intake. The MSC injections resulted in drastic reductions in the rats' alcohol consumption. A single intracerebroventricular MSC administration inhibited relapse-like drinking by up to $85 \%$ for 40 days.

It is beyond unlikely that direct brain injections would be used to treat alcohol use disorder in humans. To address this problem, researchers aggregated MSCs into smaller spheroid shapes, which reduced their size up to $75 \%$ and allowed them to be injected intravenously to reach the brain in a study conducted in rats. ${ }^{42}$ Within 48 hours of a single treatment, the rats had reduced their intake of alcohol by $90 \%$. The IV administration of antiinflammatory MSCs in human trials will be the next step to verify these results.

Alcohol research using human stem cells is also being conducted as a model system to understand the neural mechanisms of alcohol use disorder. ${ }^{43}$

Cocaine use disorder. In a grant proposal, Yadid and Popovtzer ${ }^{44}$ suggested that cocaine addiction affects neurogenesis, especially in the dentate gyrus, ventral tegmental area, nucleus accumbens, and prefrontal cortex; it damages mitochondrial RNA, brain-derived neurotrophic factor (BDNF), glutamate transporter (excitatory amino acid transporter; EAAT), and interleukin-10. MSCs have a predilection to these areas and influence neurogenesis. Currently, there are no FDAapproved medications for the safe and effective treatment of cocaine addiction. MSCs can home to pathological areas in the brain, release growth factors, and serve as cellular delivery tools in various brain disorders. Moreover, restoration of basal glutamate levels via the EAAT has been proposed as a promising target for treating cocaine dependence. Therefore, MSCs differentiated to express EAATs may have a combined long-term effect that can attenuate cocaine craving and relapse. ${ }^{44}$

Neural stem cells undergo a series of developmental processes before giving rise to newborn neurons, astrocytes, and oligodendrocytes in adult neurogenesis. During the past decade, studies of adult neurogenesis modulated by addictive drugs have highlighted the role of stem cells. These drugs have been shown to regulate the proliferation, differentiation, and survival of adult cells in different manners, which results in the varying consequences of adult neurogenesis. ${ }^{45}$ Reversal of these influences by healthy stem cells can be a worthy goal to pursue.

Opioid use disorder. Opiate medications cause a loss of newly born neural progenitors in the subgranular zone of the dentate gyrus by either modulating proliferation or interfering with differentiation and maturation. ${ }^{46}$ Opiates were the first medications shown to negatively impact neurogenesis in the adult mammalian hippocampus. ${ }^{47,48}$ The restoration of hippocampal function may positively affect the prognosis of a patient who is addicted.

Cannabinoids. Cannabinoids' influence on the brain and on stem cells is controversial. On one hand, deteriorated neurogenesis results in reduced long-term potentiation in hippocampal formation. These cellular and physiological alterations lead to decreased short-term spatial memory and increased depressionlike behaviors. ${ }^{49}$ On the other hand, there is emerging evidence that cannabinoids improve neurogenesis and CNS plasticity, at least in the adult mouse. ${ }^{50}$ Through normalization of immune function, and restoration of the brain and the body, stem cells may assist in better health and in treatment of cannabis use disorder. seem to require BDNF signaling, which suggests that BDNF holds great potential as a therapeutic agent. Cell therapies focused on correcting BDNF deficiencies in mice have had some success. ${ }^{33}$

Dysregulation of GABAergic neurons has also been implicated in depression and anxiety. Patients with major depressive disorder have reduced gamma aminobutyric acid (GABA) receptors in the parahippocampal and lateral temporal lobes. ${ }^{34}$

Ultimately, the development of differentiation protocols for serotonergic and
GABAergic neuronal populations will pave the way for examining the role of these populations in the pathogenesis of depression and anxiety, and may eventually open the door for cell-based therapies in humans. ${ }^{35}$

Studies have demonstrated a reduction in the density of pyramidal and nonpyramidal neurons in the anterior cingulate cortex of patients with schizophrenia and bipolar disorder, $^{36}$ glial reduction in the subgenual prefrontal cortex in mood disorders, ${ }^{37}$ and morphometric evidence for neuronal and glial prefrontal cell pathology in major

\section{Clinical Point}

Stem cell injections in rats bred to have high alcohol intake drastically reduced the rats' alcohol consumption 


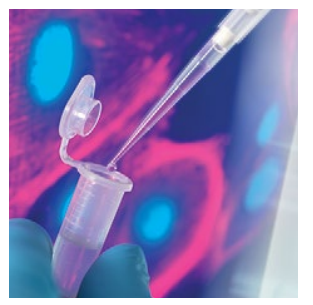

Stem cells in psychiatry

\section{Clinical Point}

iPSCs may be utilized in new pathways for research on the biology and pharmacology of major depressive disorder

\section{Related Resources}

- De Los Angeles A, Fernando MB, Hall NAL, et al. Induced pluripotent stem cells in psychiatry: an overview and critical perspective. Biol Psychiatry. 2021;90(6):362-372.

- Heider J, Vogel S, Volkmer H, et al. Human iPSC-derived glia as a tool for neuropsychiatric research and drug development. Int J Mol Sci. 2021;22(19):10254.

Drug Brand Name

Streptozotocin - Zanosar

depressive disorder. $^{38}$ The potential for stem cells to repair such pathology may be of clinical benefit to many patients.

Aside from their other suggested clinical uses, iPSCs may be utilized in new pathways for research on the biology and pharmacology of major depressive disorder. ${ }^{39}$

\section{Obsessive-compulsive disorder}

Obsessive-compulsive disorder (OCD) is often characterized by excessive behaviors related to cleanliness, including grooming, which is represented across most animal species. In mice, behaviors such as compulsive grooming and hair removal-similar to behaviors in humans with OCD or trichotillomania-are associated with a specific mutation. Chen et $\mathrm{al}^{40}$ reported that the transplantation of bone marrow stem cells into mice with this mutation (bone marrowderived microglia specifically home to the brain) rescues their pathological phenotype by repairing native neurons.

The autoimmune, inflammatory, and neurodegenerative changes that are prevalent in OCD may be remedied by stem cell treatment in a fashion described throughout this article.

\section{Other conditions}

The Box ${ }^{41-50}$ (page 39) describes a possible role for stem cells in the treatment or prevention of several types of substance use disorders.

Chronic pain is a neuropsychiatric condition that involves the immune system, inflammation, vascularization, trophic changes, and other aspects of the CNS function in addition to peripheral factors and somatic pain generators. Treatment of painful conditions with the aid of stem cells represents a large and ever-developing field that lies outside of the scope of this article. ${ }^{51}$

\section{Experimental, but promising}

It is not easy to accept revolutionary new approaches in medicine. Endless research and due diligence are needed to prove a concept and then to work out specific applications, safeguards, and limitations for any novel treatments. The stem cell terrain is poorly explored, and one needs to be careful when venturing there. Presently, the FDA appropriately sees treatment with stem cells as experimental and investigational, particularly in the mental health arena. Stem cells are not approved for treatment of any specific condition. At the same time, research and clinical practice suggest stem cell treatment may someday play a more prominent role in health care. Undoubtedly, psychiatry will eventually benefit from the knowledge and application of stem cell research and practice.

\footnotetext{
References

1. Takahashi K, Tanabe K, Ohnuki M, et al. Induction of pluripotent stem cells from adult human fibroblasts by defined factors. Cell. 2007;131(5):861-872.

2. Duncan T, Valenzuela M. Alzheimer's disease, dementia, and stem cell therapy. Stem Cell Res Ther. 2017;8(1):111.

3. Brinton RD, Wang JM. Therapeutic potential of neurogenesis for prevention and recovery from Alzheimer's disease: allopregnanolone as a proof of concept neurogenic agent. Curr Alzheimer Res. 2006;3(3):185-190.

4. Taupin P. Adult neurogenesis, neural stem cells, and Alzheimer's disease: developments, limitations, problems, and promises. Curr Alzheimer Res. 2009;6(6):461-470.
}

\section{Bottom Line}

Treatment with stem cell transplantation is experimental and not approved for any medical or psychiatric illness. However, based on our growing understanding of the function of stem cells, and preliminary research conducted mainly in animals, many neurodegenerative-, vascular-, immune-, and inflammation-based psychiatric conditions might be beneficially influenced by stem cell treatment. 
5. Taupin P. Neurogenesis, NSCs, pathogenesis, and therapies for Alzheimer's disease. Front Biosci (Schol Ed). 2011;3:178-90.

6. Kang JM, Yeon BK, Cho SJ, et al. Stem cell therapy for Alzheimer's disease: a review of recent clinical trials. J Alzheimers Dis. 2016;54(3):879-889.

7. Li M, Guo K, Ikehara S. Stem cell treatment for Alzheimer's disease. Int J Mol Sci. 2014;15(10):19226-19238.

8. Zappa VillarMF,López HanotteJ,PardoJ,etal.Mesenchymal stem cells therapy improved the streptozotocin-induced behavioral and hippocampal impairment in rats. Mol Neurobiol. 2020;57(2):600-615.

9. Lupien SJ, de Leon M, de Santi S, et al. Cortisol levels during human aging predict hippocampal atrophy and memory deficits. Nat Neurosci. 1998;1(1):69-73.

10. Iannitelli A, Quartini A, Tirassa P, et al. Schizophrenia and neurogenesis: a stem cell approach. Neurosci Biobehav Rev. 2017;80:414-442.

11. Sacco R, Cacci E, Novarino G. Neural stem cells in neuropsychiatric disorders. Curr Opin Neurobiol. 2018; 48:131-138

12. Miller ND, Kelsoe JR. Unraveling the biology of bipolar disorder using induced pluripotent stem-derived neurons. Bipolar Disord. 2017;19(7):544-551.

13. O'Shea KS, McInnis MG. Neurodevelopmental origins of bipolar disorder: iPSC models. Mol Cell Neurosci. 2016;73:63-83

14. Jacobs BM. A dangerous method? The use of induced pluripotent stem cells as a model for schizophrenia. Schizophr Res. 2015;168(1-2):563-568.

15. Liu Y, Deng W. Reverse engineering human neurodegenerative disease using pluripotent stem cell technology. Brain Res. 2016;1638(Pt A):30-41.

16. Siniscalco D, Kannan S, Semprún-Hernández N, et al. Stem cell therapy in autism: recent insights. Stem Cells Cloning. 2018;11:55-67.

17. Siniscalco D, Bradstreet JJ, Sych N, et al. Mesenchymal stem cells in treating autism: novel insights. World J Stem Cells. 2014;6(2):173-178

18. Siniscalco D, Sapone A, Cirillo A, et al. Autism spectrum disorders: is mesenchymal stem cell personalized therapy the future? J Biomed Biotechnol. 2012; 2012:480289.

19. Bradstreet JJ, Sych N, Antonucci N, et al. Efficacy of fetal stem cell transplantation in autism spectrum disorders: an open-labeled pilot study. Cell Transplant. 2014;23(Suppl 1):S105-S112.

20. Lv YT, Zhang Y, Liu M, et al. Transplantation of human cord blood mononuclear cells and umbilical cordderived mesenchymal stem cells in autism. J Transl Med. 2013;11:196.

21. Vargas DL, Nascimbene C, Krishnan C, et al. Neuroglial activation and neuroinflammation in the brain of patients with autism. Ann Neurol. 2005;57(1):67-81.

22. Wei L, Keogh CL, Whitaker VR, et al. Angiogenesis and stem cell transplantation as potential treatments of cerebral ischemic stroke. Pathophysiology. 2005;12(1): 47-62.

23. Newman MB, Willing AE, Manresa JJ, et al. Cytokines produced by cultured human umbilical cord blood (HUCB) cells: implications for brain repair. Exp Neurol. 2006;199(1):201-218.

24. Peterson DA. Umbilical cord blood cells and brain stroke injury: bringing in fresh blood to address an old problem. J Clin Invest. 2004;114(3):312-314

25. Cohly HH, Panja A. Immunological findings in autism. Int Rev Neurobiol. 2005;71:317-341.

26. Ashwood P, Van de Water J. Is autism an autoimmune disease? Autoimmun Rev. 2004;3(7-8):557-562.

27. Yagi $\mathrm{H}$, Soto-Gutierrez A, Parekkadan B, et al. Mesenchymal stem cells: mechanisms of immunomodulation and homing Cell Transplant. 2010;19(6):667-679.

28. Vaccarino FM, Urban AE, Stevens HE, et al. Annual Research Review: The promise of stem cell research for neuropsychiatric disorders. J Child Psychol Psychiatry. 2011;52(4):504-516.

29. Liu EY, Scott CT. Great expectations: autism spectrum disorder and induced pluripotent stem cell technologies. Stem Cell Rev Rep. 2014;10(2):145-150
30. Richardson-Jones JW, Craige CP, Guiard BP, et al. 5-HT1A autoreceptor levels determine vulnerability to stress and response to antidepressants. Neuron. 2010;65(1):40-52.

31. Saarelainen T, Hendolin P, Lucas G, et al. Activation of the TrkB neurotrophin receptor is induced by antidepressant drugs and is required for antidepressant-induced behavioral effects. J Neurosci. 2003;23(1):349-357.

32. Klumpers UM, Veltman DJ, Drent ML, et al. Reduced parahippocampal and lateral temporal GABAA-[11C] flumazenil binding in major depression: preliminary results. Eur J Nucl Med Mol Imaging. 2010;37(3): 565-574

33. Bremner JD, Narayan M, Anderson ER, et al. Hippocampal volume reduction in major depression. Am J Psychiatry. 2000;157(1):115-118

34. Bremner JD, Randall P, Scott TM, et al. MRI-based measurement of hippocampal volume in patients with combat-related posttraumatic stress disorder. Am J Psychiatry. 1995;152(7):973-981.

35. Vincent SL, Todtenkopf MS, Benes FM. A comparison of the density of pyramidal and non-pyramidal neurons in the anterior cingulate cortex of schizophrenics and manic depressives. Soc Neurosci Abstr. 1997;23:2199.

36. Benes FM, Kwok EW, Vincent SL, et al. A reduction of nonpyramidal cells in sector CA2 of schizophrenics and manic depressives. Biol Psychiatry. 1998;44(2): 88-97.

37. Ongür D, Drevets WC, Price JL. Glial reduction in the subgenual prefrontal cortex in mood disorders. Proc Natl Acad Sci U S A. 1998;95(22):13290-13295

38. Rajkowska G, Miguel-Hidalgo JJ, Wei J, et al. Morphometric evidence for neuronal and glial prefrontal cell pathology in major depression. Biol Psychiatry. 1999;45(9): 1085-1098.

39. Licinio J, Wong ML. Serotonergic neurons derived from induced pluripotent stem cells (iPSCs): a new pathway for research on the biology and pharmacology of major depression. Mol Psychiatry. 2016;21(1):1-2.

40. Chen SK, Tvrdik P, Peden E, et al. Hematopoietic origin of pathological grooming in Hoxb8 mutant mice. Cell. 2010;141(5):775-785.

41. Israel Y, Ezquer F, Quintanilla ME, et al. Intracerebral stem cell administration inhibits relapse-like alcohol drinking in rats. Alcohol Alcohol. 2017;52(1):1-4

42. Ezquer F, Morales P, Quintanilla ME, et al. Intravenous administration of anti-inflammatory mesenchymal stem cell spheroids reduces chronic alcohol intake and abolishes binge-drinking. Sci Rep. 2018;8(1):4325.

43. Scarnati MS, Halikere A, Pang ZP. Using human stem cells as a model system to understand the neural mechanisms of alcohol use disorders: current status and outlook. Alcohol. 2019;74:83-93.

44. Yadid GM, Popovtzer R. Nanoparticle-mesenchymal stem cell conjugates for cell therapy in drug addiction. NIH grant application. 2017.

45. Xu C, Loh HH, Law PY. Effects of addictive drugs on adult neural stem/progenitor cells. Cell Mol Life Sci. 2016;73(2):327-348.

46. Dholakiya SL, Aliberti A, Barile FA. Morphine sulfate concomitantly decreases neuronal differentiation and opioid receptor expression in mouse embryonic stem cells. Toxicol Lett. 2016;247:45-55.

47. Zhang Y, Loh HH, Law PY. Effect of opioid on adult hippocampal neurogenesis. Scientific World Journal. 2016;2016:2601264.

48. Bortolotto V, Grilli M. Opiate analgesics as negative modulators of adult hippocampal neurogenesis: potential implications in clinical practice. Front Pharmacol. 2017 8:254.

49. Galve-Roperh I, Chiurchiù V, Díaz-Alonso J, et al. Cannabinoid receptor signaling in progenitor/stem cell proliferation and differentiation. Prog Lipid Res. 2013; 52(4):633-650

50. Zimmermann T, Maroso M, Beer A, et al. Neural stem cell lineage-specific cannabinoid type-1 receptor regulates neurogenesis and plasticity in the adult mouse hippocampus. Cereb Cortex. 2018;28(12):4454-4471.

51. Ren J, Liu N, Sun N, et al. Mesenchymal stem cells and their exosomes: promising therapies for chronic pain. Curr Stem Cell Res Ther. 2019;14(8):644-653.
Current

$\nabla$ MDedge.com/psychiatry

\section{Clinical Point}

The FDA appropriately

sees treatment

with stem cells as

experimental and

investigational,

especially in the

mental health arena

Current Psychiatry

Vol. 20, No. 12 